\title{
Incidence, patterns, risk factors, and histopathological findings of liver injury in coronavirus disease 2019 (COVID-19): a scoping review
}

\author{
Taha Bin Arif *, Saad Khalid, Mishal S Siddiqui, Harmla Hussain, Hassan Sohail
}

\section{A B S T R A C T}

Background: Coronavirus disease 2019 (COVID-19) exhibits many extrapulmonary manifestations, including liver injury. This scoping review aimed to provide insight into the incidence, patterns, risk factors, histopathological findings, and relationship with disease severity of COVID-19-associated liver injury. Furthermore, we identified existing gaps in the research on the hepatic manifestations of COVID-19 and highlighted areas for future investigations.

Methods: A scoping review was conducted following the methodological framework suggested by Arksey and O'Mallay. Five online databases, along with grey literature, were searched for articles published until 22 May 2020, and we included 62 articles in the review. The research domains, methodological characteristics, and key conclusions were included in the analysis.

Results: Retrospective observational studies comprised more than one-third (41.9\%) of the included publications, and $77.8 \%$ were conducted on living patients. The incidence of liver injury varied widely across the studies (4.8\%-78\%), and liver injury was frequently associated with severe COVID-19.

This article was published on 30 Oct 2020 at www.hkmj.org.
We identified the following risk factors for liver injury: male sex, lymphopoenia, gastrointestinal involvement, old age, increased neutrophil count, and the use of hepatotoxic drugs. Histopathological findings indicate that COVID-19 has direct cytopathic effects and causes liver function test derangements secondary to inflammation, hypoxia, and vascular insult.

Conclusions: Liver injury following COVID-19 infection is common and primarily hepatocellular, with a greater elevation of aspartate aminotransferase than of alanine aminotransferase. However, the evidence regarding hepatic failure secondary to COVID-19 is insufficient. Standardised criteria to diagnose liver injury need to be devised. Current use of hepatotoxic drugs necessitates close monitoring of liver function.

\section{Hong Kong Med J 2020;26:198-209 \\ https://doi.org/10.12809/hkmj208732}

T Bin Arif *, MB, BS

$S$ Khalid, MB, BS

MS Siddiqui, MB, BS

H Hussain, MB, BS

H Sohail, MB, BS

Department of Internal Medicine, Dow University of Health Sciences, Karachi, Pakistan

* Corresponding author: tahaarif20@yahoo.com

\section{Introduction}

The coronavirus disease 2019 (COVID-19) pandemic has spread to 213 countries and territories, posing a severe threat to public healthcare systems worldwide. As of 29 May 2020, the total number of confirmed cases has surged to 5701337, with 357688 deaths recorded worldwide. ${ }^{1}$ Although multiple pharmacological agents are being evaluated, no beneficial, targeted drug or vaccine has been discovered to date, and the number of cases is rising daily. The causative agent of COVID-19 is severe acute respiratory syndrome coronavirus 2 (SARS-CoV-2), which is believed to be transmitted through respiratory droplets and person-to-person contact. However, evidence of viral RNA in the faeces of COVID-19 patients also suggests the possibility of faecal-oral transmission., ${ }^{2,3}$ The disease typically presents with viral pneumonia-like symptoms of fever, dry cough, shortness of breath, and fatigue. Nevertheless, gastrointestinal symptoms like diarrhoea, vomiting, and abdominal pain have also been reported. ${ }^{4}$

Although coronavirus mainly targets the respiratory system, it also exhibits many extrapulmonary manifestations. Sepsis, acute cardiac injury, multiple organ failure, and alkalosis are some of the critical complications that have been observed in patients who die of COVID-19. ${ }^{5}$ Several studies have acknowledged the presence of liver injury in patients with COVID-19, mainly indicated by abnormal liver function tests (LFTs) ${ }^{6,7}$ The exact pathophysiology behind the LFT derangements is unknown. It has been suggested that SARS-CoV-2 causes liver injury either via direct viral insult or through an inflammatory cytokine storm. ${ }^{8}$ Other potential mechanisms, such as drug-induced 
hepatotoxicity and hypoxic injury, have also been implicated.

Given the rampant nature of SARS-CoV-2 and its repercussions on human health, the research community has responded expeditiously to the new virus, and studies regarding its systemic involvement are continuouslybeing published. We have conducted a scoping review to summarise all articles published regarding hepatic damage in this setting. In this review, we aim to provide evidence of the incidence, patterns, risk factors, and histopathological findings of liver injury in COVID-19 and its association with the severity of disease. Furthermore, we highlight hepatotoxicity in patients with COVID-19 who are treated with antiviral (lopinavir/ritonavir) or antimalarial (hydroxychloroquine) drugs. We identify the existing gaps in current knowledge regarding the topic and provide recommendations for further research. This will help healthcare providers to identify hepatic complications during the pandemic.

\section{Methods}

\section{Study design}

A scoping review was conducted following the methodological framework of Arksey and O'Malley ${ }^{9}$ by taking the following steps: (a) identification of a definite research objective and search strategy; (b) identification and screening of research articles; (c) selection of research articles according to pre-defined eligibility criteria; (d) extraction and charting of data, and (e) reporting, summarising, and discussing the results.

\section{Literature search strategies}

The reviewed literature was identified by searching five online databases (PubMed, Google Scholar, Scopus, Wiley, and ScienceDirect) without any language restriction from 1 January 2020 to 22 May 2020. Grey literature was also searched in medRxiv and bioRxiv. Moreover, the reference lists of all identified articles were searched for additional sources. A variety of keywords were employed, according to the following search string: "liver injury" OR "hepatic damage" OR "liver functional abnormality" OR "cirrhosis" OR "decompensated liver disease" OR "acute liver failure" OR "chronic liver failure" OR "acute on chronic liver failure" AND "COVID-19" OR "SARS-CoV-2" OR "coronavirus disease". The full electronic search strategy is provided in the online supplementary Appendix.

\section{Identification, screening, and selection of relevant studies}

We aimed to summarise all of the scientific literature demonstrating liver dysfunction in COVID-19 and to identify the gaps in knowledge regarding hepatic

\section{9 冠狀病毒病（COVID-19）造成肝損傷的 發生率、模式、危險因素和組織病理學發現： 範圍性綜述}

Taha Bin Arif, Saad Khalid, Mishal S Siddiqui, Harmla Hussain, Hassan Sohail

背景：2019冠狀病毒病（COVID-19）具有許多肺外表現, 當中包 括肝損傷。本範圍性綜述旨在探入了解與COVID-19相關肝損傷的發 生率、模式、危險因素、組織病理學發現, 以及與疾病重度關係。此 外, 我們在文獻中找出COVID-19肝表現和需要進一步研究領域的存 在差距。

方法：根據Arksey和O'Mallay建議的方法框架進行範圍性審查。搜索 了五個在線數據庫以及灰色文獻，以查找直到2020年5月22日為止發 表的文章。共納入 62 篇文章, 分析它們的研究領域、方法學特徵和主 要結論。

結果：62篇文章中, 超過三分之一為回顧性觀察研究 $(41.9 \%)$, 有 關倖存患者的研究佔 $77.8 \%$ 。這些研究當中, 肝損傷的發生率存在很 大差異 ( $4.8 \%-78 \%)$ ，並且肝損傷通常與嚴重COVID-19相關。研究 也確定以下肝損傷的危險因素, 包括男性、淋巴細胞、胃腸道受累、 老年、中性粒細胞數目增加以及使用肝毒性藥物。組織病理學發現顯 示COVID-19會直接影響細胞病變, 引起繼發於炎症、缺氧和血管損 傷的紊亂。

結論：COVID-19常造成肝損傷，主要是肝細胞，與丙氨酸轉氨酶相 比, 天冬氨酸轉氨酶的上升幅度更大。不過, 有關COVID-19繼發肝 衰竭的證據不足。應當制定診斷肝損傷的標準化標準。使用肝毒性藥 物時必須對肝功能進行密切監測。

damage in SARS-CoV-2 infection for further research. Three researchers (TBA, SK, and MSS) independently searched through the literature, and all sets of literature were then compared. Disagreements on the inclusion or exclusion of literature were resolved through discussion or, if necessary, by including a fourth researcher $(\mathrm{HH})$ to make the final decision. Articles were screened according to pre-defined eligibility criteria. The inclusion criteria were as follows: (1) study design: retrospective observational study, retrospective cohort study, retrospective descriptive study, prospective observational study, prospective case-cohort, cross-sectional, case report, case series, or meta-analysis; (2) language: studies published in English only; (3) publication status: preprints and published articles; (4) dates considered: studies published from 1 January 2020 to 22 May 2020; and (5) all relevant papers describing functional abnormalities of the liver in COVID-19. The exclusion criteria were as follows: (1) language: articles published in any language other than English; (2) study design: review article, editorial, letter to the editor, correspondence, or commentary; and (3) studies conducted on patients who had undergone organ transplants. Duplicate articles were excluded. Ultimately, 62 articles were included in this review conducted in accordance with the Preferred 


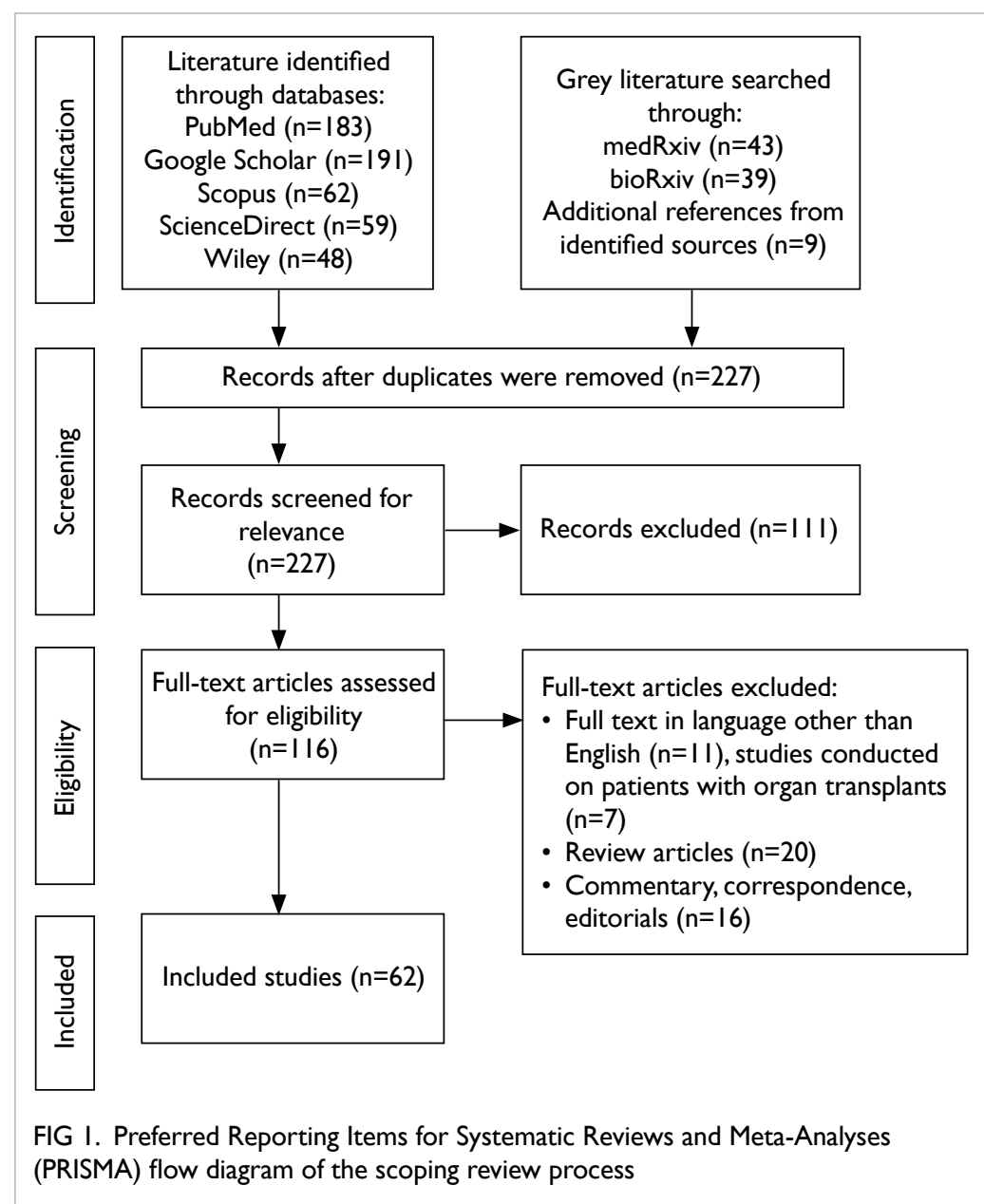

Reporting Items for Systematic Reviews and MetaAnalyses extension for scoping reviews (Fig 1). ${ }^{10}$

\section{Data extraction and charting from included studies}

After article selection, data were extracted and recorded on a pre-designed datasheet. The extracted data included the article's title, study design, study setting, study population, sample size, research domain, and key conclusions.

\section{Summarising the studies}

The articles that assessed liver injury in patients with COVID-19 belong to two categories: (a) studies that employed pre-defined clinical criteria for liver injury in COVID-19, and (b) studies that did not employ any pre-defined criteria and only reported LFT derangements in COVID-19. Based on the primary research objectives, each article was classified into one of the following main research domains: incidence of liver injury, patterns of liver injury, and risk factors for liver injury in COVID-19, associations of liver injury or underlying liver disease (eg, chronic liver disease) with the severity of COVID-19, drug-induced liver injury in COVID-19, and histopathological findings of liver injury in COVID-19. The methodological characteristics (study design, study setting, type of population, and sample size) of all studies were also analysed.

\section{Results and discussion}

\section{Characteristics of studies}

A total of 62 articles were included in this scoping review, among which 10 were preprints, and 52 were published in peer-reviewed journals, including The Lancet and Journal of the American Medical Association. About 23 studies (16 retrospective observational, 2 retrospective cohort, 1 retrospective descriptive, 1 prospective observational, 1 prospective case-cohort, 1 cross-sectional, and 1 meta-analysis) documented the incidence of liver injury in COVID-19. ${ }^{11-33}$ Around half of the eligible studies $(\mathrm{n}=29,46.8 \%)$ showed an association between the severity of COVID-19 and the degree of liver injury. ${ }^{11-17,19-23,28,29,34-48}$

Out of the 27 studies assessing liver injury in COVID-19, 44.4\% ( $\mathrm{n}=12)$ had pre-defined clinical criteria for liver injury, whereas $55.6 \%(\mathrm{n}=15)$ did not have any specific pre-defined criteria. The details of these studies are given below. The studies predominantly depicted significant elevation of aspartate aminotransferase (AST) than of alanine aminotransferase (ALT) in case of liver injury, which was found to be proportional to the severity of COVID-19.

Fewer studies $(\mathrm{n}=6)$ mentioned any histopathological findings of liver injury in patients with COVID-19, but the most common findings mentioned were mild sinusoidal dilatation, microvesicular steatosis, and minimal lymphocytic infiltration. ${ }^{36,49-53}$ Eight studies assessed the impact of drugs on potential liver damage. Half of those studies $(n=4,50 \%)$ concluded that the use of lopinavir/ritonavir increases the odds of liver injury. Other drugs described as having the potential to cause hepatotoxicity in COVID-19 included hydroxychloroquine $(\mathrm{n}=1,12.5 \%)$, tocilizumab $(\mathrm{n}=2$, $25 \%)$, and remdesivir $(\mathrm{n}=1,12.5 \%) .12,18,54-59$

The methodological characteristics of the finalised studies were also analysed. The largest number of the studies were retrospective observational studies $(\mathrm{n}=26,41.9 \%)$, followed by meta-analyses $(n=10,16.1 \%)$, case reports $(n=9$, $14.5 \%)$, case series $(n=7,11.3 \%)$, prospective observational studies $(\mathrm{n}=3,4.8 \%)$, and others (Table 1). All studies except for meta-analyses, case reports, and series included a targeted population. Among the 36 articles with a targeted population, more than three-quarters $(n=28,77.8 \%)$ were conducted only on living patients with COVID-19, whereas the remainder $(n=8,22.2 \%)$ included patients who died. Of the finalised studies, $38.9 \%$ $(n=14)$ had sample sizes of 5 to 60 . The included 
studies' methodological characteristics are given in Table 1.

\section{Incidence of liver injury in patients with COVID-19}

Several observational studies documenting the clinical characteristics of patients with COVID-19 have reported liver injury. ${ }^{11-33}$ They have mentioned liver enzyme elevation without commenting on the clinical signs of hepatic dysfunction, which include hepatomegaly, ascites, and jaundice. ${ }^{11-30,32}$ The incidence of liver injury has varied widely across studies, from $4.8 \%$ to a striking $78 \%{ }^{11-33}$ However, the term 'liver injury' has not been defined uniformly. The definitions used in various studies have ranged from slight transaminasaemia to enzyme elevation more than 3 times higher than the upper limit of normal (Table $2^{11,12,16-18,20,22-24,31-33}$ ). Additionally, several studies did not establish any clinical or laboratory criteria to define liver injury (Table $3^{13-15,19,21,25-29,34,35,37,46,60}$. Many studies failed to mention the date on which LFTs were performed, thus creating non-uniformity in their reported values. Case reports have identified the presence of liver injury across the entire age spectrum, ranging from 55 days to 65 years. ${ }^{50,57,58,61,62}$

\section{Pathogenesis of liver dysfunction in COVID-19}

The pathogenesis of liver involvement in COVID-19 infection is assumed to be multifactorial. However, none of the available hypotheses provide a complete explanation, and further investigation is required not only to understand the mechanism but also to formulate appropriate management plans. Figure 2 illustrates the possible mechanisms of hepatic dysfunction in COVID-19.

\section{Direct viral invasion}

The proposed receptor for the virus, angiotensinconverting enzyme 2 receptor (ACE2R), has been found only sparsely in hepatocytes. Chai et $\mathrm{al}^{63}$ demonstrated ACE2 expression in $2.6 \%$ of hepatocytes, whereas up to $59.7 \%$ of cholangiocytes expressed ACE2R. Seow et $\mathrm{al}^{64}$ also revealed the presence of ACE2 in liver progenitor cells, especially those destined to become cholangiocytes. These findings imply direct invasion of cholangiocytes and progenitor cells, thus resulting in necrosis and impaired regeneration of cholangiocytes. The tight junctions between cholangiocytes also seem to be altered during COVID-19 infection, which may be responsible for the observed cholestasis in patients. ${ }^{65}$ Significant necrosis of and rapid viral replication within cholangiocytes has also been observed by Zhao et $\mathrm{al}^{65}$ in a human liver ductal organoid model.

However, Zhou et $\mathrm{al}^{66}$ argued against this proposed mechanism by highlighting that ACE2Rs on cholangiocytes are confined to the apical surface, from where viral invasion is unlikely. Furthermore, the hepatic pattern of LFT elevation fails to explain this possible ductal pathology. Hepatocytes also express the protein furin, which may play a role in liver damage upon entry of the virus into the cells.

\section{Hypoxia}

Decreased oxygen saturation has been a feature of COVID-19 pneumonia, and this may result in hypoxic injury to multiple organs, including the liver. ${ }^{67}$

\section{High positive end expiratory pressure}

High values of positive end expiratory pressure used during mechanical ventilation in severe patients may result in hepatic congestion by increasing the pressure on the right atrium and thereby impeding venous return. However, the presence of comparable liver functional abnormalities in patients without ventilation renders that assumption inconclusive. ${ }^{68}$

\section{Systemic inflammation and cytokine storm}

An inflammatory response to the virus may lead to persistent leukocytic activation and the release of many mediators responsible for cellular injury. The involvement of a cytokine storm in liver damage has been supported by patients' elevated levels of interleukins 2, 6, and 10, interferon-gamma, serum ferritin, and C-reactive protein. ${ }^{17}$

TABLE I. Methodological characteristics of studies

\begin{tabular}{llc}
\hline $\begin{array}{l}\text { Methodological characteristics } \\
\text { of studies }\end{array}$ & Categories & No. (\%) \\
\hline Study design & Prospective case-cohort & $1(1.6 \%)$ \\
& Retrospective descriptive & $2(3.2 \%)$ \\
& Case series & $7(11.3 \%)$ \\
& Case report & $9(14.5 \%)$ \\
& Meta-analysis & $10(16.1 \%)$ \\
& Retrospective observational & $26(41.9 \%)$ \\
& Cross-sectional & $1(1.6 \%)$ \\
& Prospective observational & $3(4.8 \%)$ \\
\hline $\begin{array}{l}\text { Type of study population } \\
\text { (excluding meta-analyses and }\end{array}$ & Retrospective cohort & $3(4.8 \%)$ \\
case reports/series) & potal studies with a targeted & 36 \\
& Patients (alive) & $28(77.8 \%)$ \\
\hline $\begin{array}{l}\text { Sample size (excluding meta- } \\
\text { analyses and case reports/series) }\end{array}$ & $5-60$ & $8(22.2 \%)$ \\
& $61-150$ & $14(38.9 \%)$ \\
& $151-300$ & $10(27.8 \%)$ \\
& $301-500$ & $4(11.1 \%)$ \\
& $>500$ & $5(13.9 \%)$ \\
\hline
\end{tabular}


TABLE 2. Summary of COVID-19 studies with pre-defined criteria for liver injury/hepatic dysfunction

\begin{tabular}{|c|c|c|c|c|c|c|c|c|}
\hline Author & Study type & $\begin{array}{l}\text { Study } \\
\text { location }\end{array}$ & $\begin{array}{l}\text { Sample } \\
\text { size }\end{array}$ & $\begin{array}{l}\text { Clinical criteria for } \\
\text { liver injury/hepatic } \\
\text { dysfunction }\end{array}$ & $\begin{array}{l}\text { Incidence of } \\
\text { liver injury/ } \\
\text { hepatic } \\
\text { dysfunction, } \\
\%(n)\end{array}$ & $\begin{array}{l}\text { Liver function tests } \\
\text { (median [IQR] or } \\
\text { mean [SD]) }\end{array}$ & $\begin{array}{l}\text { Risk factors } \\
\text { of liver injury/ } \\
\text { hepatic } \\
\text { dysfunction }\end{array}$ & Study notes \\
\hline Cai et $\mathrm{al}^{12}$ & $\begin{array}{l}\text { Cross- } \\
\text { sectional } \\
\text { study }\end{array}$ & $\begin{array}{l}\text { Shenzhen, } \\
\text { China }\end{array}$ & 417 & $\begin{array}{l}\text { ALT and/or AST } 3 \text { times } \\
\text { ULN; ALP, GGT and/or } \\
\text { TBIL } 2 \text { times ULN }\end{array}$ & $21.5 \%(90)$ & $\begin{array}{l}\mathrm{ALT}=21(15-31)^{\star} \\
\mathrm{AST}=26.5(21-35)^{\star} \\
\mathrm{TBIL}=10.9(8.3-16.3)^{\star}\end{array}$ & $\begin{array}{l}\text { Use of lopinavir/ } \\
\text { ritonavir }\end{array}$ & $\begin{array}{l}\text { Patients with liver injury } \\
\left(\mathrm{ALT}=47[38-65.2]^{*} \mathrm{U} / \mathrm{L} ;\right. \\
\mathrm{AST}=47.2[30.9-63.8]^{*} \mathrm{U} / \mathrm{L} \text { ) } \\
\text { were at increased risk of } \\
\text { severe disease } \\
\text { Liver injury was mainly } \\
\text { caused by medications } \\
\text { used during hospitalisation }\end{array}$ \\
\hline Cai et $\mathrm{al}^{16}$ & $\begin{array}{l}\text { Retrospective } \\
\text { observational }\end{array}$ & $\begin{array}{l}\text { Shenzhen, } \\
\text { China }\end{array}$ & 298 & $\begin{array}{l}\text { ALT and/or AST } 3 \text { times } \\
\text { ULN; GGT and/or TBIL } \\
2 \text { times ULN }\end{array}$ & $14.8 \%(44)$ & $\begin{array}{l}\mathrm{ALT}=21(15-30.7)^{\star} \\
\mathrm{AST}=27.3(22-36.3)^{\star} \\
\mathrm{TBIL}=10.9(8.2-15.9)^{\star}\end{array}$ & $\begin{array}{l}\text { Use of drugs such } \\
\text { as lopinavir and } \\
\text { ritonavir }\end{array}$ & $\begin{array}{l}\text { Liver injury was mainly } \\
\text { observed in severe patients } \\
\text { (ALT = } 42[28-78]^{\star} \mathrm{U} / \mathrm{L} ; \\
\left.\mathrm{AST}=38[23-65.5]^{*} \mathrm{U} / \mathrm{L}\right) \\
\text { and is likely to be an } \\
\text { outcome of drugs used to } \\
\text { treat the infection }\end{array}$ \\
\hline Fan et $a^{18}$ & $\begin{array}{l}\text { Retrospective } \\
\text { observational }\end{array}$ & $\begin{array}{l}\text { Shanghai, } \\
\text { China }\end{array}$ & 148 & $\begin{array}{l}\text { Liver function tests } \\
\text { above ULN }\end{array}$ & $37.2 \%(55)$ & - & $\begin{array}{l}\text { Male sex, } \\
\text { elevated CRP/ } \\
\text { PCT, use of } \\
\text { lopinavir/ritonavir }\end{array}$ & $\begin{array}{l}\text { COVID-19-related liver } \\
\text { injury may be relatively mild } \\
\text { Liver injury may be related } \\
\text { to the use of lopinavir/ } \\
\text { ritonavir }\end{array}$ \\
\hline Lei et $\mathrm{al}^{22}$ & $\begin{array}{l}\text { Retrospective } \\
\text { cohort }\end{array}$ & $\begin{array}{l}\text { Hubei, } \\
\text { China }\end{array}$ & 5771 & ALT 3 times ULN & $6.2 \%(357)$ & $\begin{array}{l}\mathrm{ALT}=24.0(15.1-39.0)^{\star} \\
\mathrm{AST}=24.0(17.0-35.0)^{\star} \\
\mathrm{TBIL}=10.4(7.9-14.1)^{\star} \\
\mathrm{ALP}=64.0(51.0-83.0)^{\star}\end{array}$ & $\begin{array}{l}\text { Male sex, } \\
\text { systemic } \\
\text { corticosteroids, } \\
\text { increased } \\
\text { neutrophil count, } \\
\text { decreased } \\
\text { lymphocyte count, } \\
\text { and fever }\end{array}$ & $\begin{array}{l}\text { Patients with severe disease } \\
\text { had higher AST levels ( } 31.0 \\
{[21.0-46.0]^{*} \mathrm{U} / \mathrm{L} \text { ) compared }} \\
\text { with those who had non- } \\
\text { severe disease ( } 22.0 \text { [17.0- } \\
\left.31.0]^{*} \mathrm{U} / \mathrm{L}\right) \text {. Elevated AST } \\
\text { was associated with high } \\
\text { mortality risk }\end{array}$ \\
\hline $\begin{array}{l}\text { Hajifathalian } \\
\text { et } \mathrm{al}^{23}\end{array}$ & $\begin{array}{l}\text { Retrospective } \\
\text { observational }\end{array}$ & $\begin{array}{l}\text { New York, } \\
\text { United } \\
\text { States }\end{array}$ & 1059 & $\begin{array}{l}\text { Elevated ALT, AST, ALP, } \\
\text { or TBIL }\end{array}$ & $62 \%(657)$ & $\begin{array}{l}\mathrm{ALT}=49.5(64.9) \dagger \\
\mathrm{AST}=59.5(78.5) \dagger \\
\mathrm{TBIL}=11.97(10.26) \dagger \\
\mathrm{ALP}=88.1(74.1) \dagger\end{array}$ & Old age & $\begin{array}{l}\text { In-patients had a } \\
\text { statistically significant } \\
\text { increase in AST compared } \\
\text { with out-patients (62.1 } \\
\text { [82.1]† vs } 46.4 \text { [56.2]† U/L) } \\
\text { Liver injury at presentation } \\
\text { was an independent } \\
\text { predictor of poor clinical } \\
\text { outcomes (ICU admission } \\
\text { or death) }\end{array}$ \\
\hline Jin et $a^{24}$ & $\begin{array}{l}\text { Retrospective } \\
\text { observational }\end{array}$ & $\begin{array}{l}\text { Zhejiang, } \\
\text { China }\end{array}$ & 651 & $\begin{array}{l}\text { AST }<40 \mathrm{U} / \mathrm{L} \\
\mathrm{ALT}<50 \mathrm{U} / \mathrm{L}\end{array}$ & $9.8 \%(64)$ & - & Gl symptoms & $\begin{array}{l}\text { Incidence of liver injury in } \\
\text { patients with Gl symptoms } \\
\text { was higher than that in } \\
\text { those without Gl symptoms } \\
(17.57 \% \text { vs } 8.84 \%) \text {. AST } \\
\text { was significantly higher in } \\
\text { patients with Gl symptoms }\end{array}$ \\
\hline Xie et $\mathrm{a}^{17}$ & $\begin{array}{l}\text { Retrospective } \\
\text { observational }\end{array}$ & $\begin{array}{l}\text { Wuhan. } \\
\text { China }\end{array}$ & 79 & $\begin{array}{l}\text { Elevated AST, ALT, or } \\
\text { TBIL }\end{array}$ & $36.7 \%(29)$ & $\begin{array}{l}\mathrm{ALT}=34(18-67)^{\star} \\
\mathrm{AST}=30(23-50)^{\star} \\
\mathrm{TBIL}=13.6(8.8-17.6)^{\star}\end{array}$ & $\begin{array}{l}\text { Male sex, } \\
\text { increased WBC } \\
\text { and neutrophil } \\
\text { count, elevated } \\
\text { CRP, lung lesions } \\
\text { on CT }\end{array}$ & $\begin{array}{l}\text { Severe lung lesions on CT } \\
\text { may be related to higher } \\
\text { incidence of liver injury. In } \\
\text { patients with liver injury, } \\
\text { GGT and ALP levels were } \\
\text { also elevated compared } \\
\text { with those in patients } \\
\text { without liver injury }\end{array}$ \\
\hline Wang et $\mathrm{al}^{11}$ & $\begin{array}{l}\text { Retrospective } \\
\text { observational }\end{array}$ & $\begin{array}{l}\text { Beijing } \\
\text { and Anhui, } \\
\text { China }\end{array}$ & 156 & $\begin{array}{l}\text { ALT or AST }<40 \mathrm{U} / \mathrm{L} \text { for } \\
>7 \text { days }\end{array}$ & $41 \%(64)$ & - & - & $\begin{array}{l}\text { Liver enzyme abnormality } \\
\text { was associated with severe } \\
\text { disease, higher A-aDO2 and } \\
\text { GGT, and lower albumin } \\
\text { and lymphocytes }\end{array}$ \\
\hline
\end{tabular}

Abbreviations: A-aDO2 = alveolar-arterial oxygen tension difference; $A L P=$ alkaline phosphatase $(U / L) ; A L T=$ alanine aminotransferase $(U / L)$;

AST = aspartate aminotransferase (U/L); COVID-I9 = coronavirus disease 20I9; CRP = C-reactive protein; CT = computed tomography; GGT = gammaglutamyl transferase (U/L); GI = gastrointestinal; ICU = intensive care unit; IQR = interquartile range; PCT = procalcitonin; SD = standard deviation; $\mathrm{TBIL}=$ total bilirubin $(\mu \mathrm{mol} / \mathrm{L}) ; \mathrm{ULN}=$ upper limit of normal; WBC = white blood cell

* Data are shown as median and IQR (in parentheses)

† Data are shown as mean and SD (in parentheses) 
TABLE 2. (cont'd)

\begin{tabular}{|c|c|c|c|c|c|c|c|c|}
\hline Author & Study type & $\begin{array}{l}\text { Study } \\
\text { location }\end{array}$ & $\begin{array}{l}\text { Sample } \\
\text { size }\end{array}$ & $\begin{array}{l}\text { Clinical criteria for } \\
\text { liver injury/hepatic } \\
\text { dysfunction }\end{array}$ & $\begin{array}{l}\text { Incidence of } \\
\text { liver injury/ } \\
\text { hepatic } \\
\text { dysfunction, } \\
\% \text { (n) }\end{array}$ & $\begin{array}{l}\text { Liver function tests } \\
\text { (median [IQR] or } \\
\text { mean [SD]) }\end{array}$ & $\begin{array}{l}\text { Risk factors } \\
\text { of liver injury/ } \\
\text { hepatic } \\
\text { dysfunction }\end{array}$ & Study notes \\
\hline Bloom et $\mathrm{al}^{20}$ & $\begin{array}{l}\text { Prospective } \\
\text { observational }\end{array}$ & $\begin{array}{l}\text { Massa- } \\
\text { chusetts, } \\
\text { United } \\
\text { States }\end{array}$ & 60 & $\begin{array}{l}\text { Grade 0: } \\
\text { AST }<40 \mathrm{U} / \mathrm{L} \text { or } \\
\text { ALT }<55 \mathrm{U} / \mathrm{L} \text { (male); } \\
\text { AST }<32 \mathrm{U} / \mathrm{L} \text { or } \\
\text { ALT }<33 \mathrm{U} / \mathrm{L} \text { (female) } \\
\text { Grade } 1: \\
\text { AST }=41-120 \mathrm{U} / \mathrm{L} \text { or } \\
\mathrm{ALT}=34-99 \mathrm{U} / \mathrm{L} \text { (male); } \\
\text { AST }=33-96 \mathrm{U} / \mathrm{L} \\
\text { or ALT }=34-99 \mathrm{U} / \mathrm{L} \\
\text { (female) } \\
\text { Grade } 2: \\
\text { AST }=121-200 \mathrm{U} / \mathrm{L} \\
\text { or ALT }=166-275 \mathrm{U} / \mathrm{L} \\
\text { (male); } \\
\text { AST }=97-160 \mathrm{U} / \mathrm{L} \text { or } \\
\text { ALT }=100-165 \mathrm{U} / \mathrm{L} \\
\text { (female) } \\
\text { Grade } 3-4: \\
\text { AST }>201 \mathrm{U} / \mathrm{L} \text { or } \\
\text { ALT }>276 \mathrm{U} / \mathrm{L} \text { (male); } \\
\text { AST }>161 \mathrm{U} / \mathrm{L} \text { or } \\
\text { ALT }>166 \mathrm{U} / \mathrm{L} \text { (female) }\end{array}$ & $\begin{array}{l}\text { Grade 0: } 7 \%(4) \\
\text { Grade 1: } 54 \% \\
\text { (32) } \\
\text { Grade 2: } 22 \% \\
\text { (13) } \\
\text { Grade 3-4: } 17 \% \\
\text { (10) }\end{array}$ & $\begin{array}{l}\text { ALT }=39(24) \dagger \\
\text { AST }=55(37) \dagger \\
\text { TBIL }=0.9(2.2) \dagger\end{array}$ & Severe disease & $\begin{array}{l}\text { AST in patients requiring } \\
\text { intubation was significantly } \\
\text { higher compared with } \\
\text { the level in those without } \\
\text { intubation (364 [572] vs } 77 \\
\text { [54])† }\end{array}$ \\
\hline Chen et $\mathrm{al}^{31}$ & $\begin{array}{l}\text { Retrospective } \\
\text { observational }\end{array}$ & $\begin{array}{l}\text { Wuhan, } \\
\text { China }\end{array}$ & 21 & $\begin{array}{l}\text { Jaundice with TBIL of } \\
\geq 3 \mathrm{mg} / \mathrm{dL} \text { and ALT } \\
5 \text { times ULN and/or } \\
\text { ALP } 2 \text { times ULN }\end{array}$ & $4.8 \%(1)$ & $\begin{array}{l}\mathrm{ALT}=26(16-42)^{\star} \\
\mathrm{AST}=27(21-47)^{\star} \\
\mathrm{TBIL}=8.8(6.8-10.3)^{\star}\end{array}$ & - & $\begin{array}{l}\text { ALT and AST were } \\
\text { significantly higher in severe } \\
\text { cases }\left(A L T=42[32.5-50]^{*} \mathrm{U} / \mathrm{L} \text {; }\right. \\
\left.\text { AST }=47[28-74.5]^{\star} \mathrm{U} / \mathrm{L}\right) \\
\text { than moderate cases }\end{array}$ \\
\hline Qi et $\mathrm{al}^{32}$ & $\begin{array}{l}\text { Multicentre } \\
\text { cohort }\end{array}$ & China & 70 & $\begin{array}{l}\text { ALT }<40 \mathrm{U} / \mathrm{L}, \\
\text { AST }<40 \mathrm{U} / \mathrm{L}, \\
\mathrm{TBIL}<17.1 \mu \mathrm{mol} / \mathrm{L}\end{array}$ & $45.7 \%(32)$ & - & $\begin{array}{l}\text { Longer time from } \\
\text { onset of illness to } \\
\text { admission }\end{array}$ & - \\
\hline $\begin{array}{l}\text { Zheng et } \\
\mathrm{al}^{33}\end{array}$ & $\begin{array}{l}\text { Retrospective } \\
\text { observational }\end{array}$ & $\begin{array}{l}\text { Hangzhou, } \\
\text { China }\end{array}$ & 34 & $\begin{array}{l}\text { ALT } 2 \text { times ULN or } \\
\text { conjugated bilirubin } \\
\text { or combined increase } \\
\text { in AST, ALP, and TBIL } \\
\text { provided that one of } \\
\text { them was }>2 \text { times ULN }\end{array}$ & $41.2 \%(14)$ & $\begin{array}{l}\mathrm{ALT}=20(20-30)^{\star} \\
\mathrm{TBIL}=12(7.6-18.6)^{\star}\end{array}$ & $\begin{array}{l}\text { Receiving invasive } \\
\text { mechanical } \\
\text { ventilation }\end{array}$ & - \\
\hline
\end{tabular}

\section{Endothelial dysfunction}

Liver dysfunction may occur secondary to vascular pathology, resulting in endotheliitis, coagulopathy, thrombus formation, and ischaemic parenchymal necrosis. Angiotensin-converting enzyme 2 receptors are expressed on the endothelium, making it susceptible to viral invasion, which leads to the recruitment of inflammatory cells and elaboration of inflammatory cytokines. The immune response may also exacerbate the damage. ${ }^{69}$

\section{Drug-induced hepatotoxicity}

A number of drugs currently in use have hepatotoxic potential, which might be further exaggerated in the setting of chronic liver disease (CLD). ${ }^{12,56,58}$ The mechanisms for individual drugs are not clearly defined.

\section{Patterns of liver injury in COVID-19}

The patterns of liver injury in COVID-19 patients include both reversible dysfunction and irreversible injury as a component of multiorgan failure in terminally ill patients. ${ }^{40,57-59,61,62}$ However, hepatic dysfunction in COVID-19 cases is usually mild, and deranged LFTs tend to recover within a few days after discharge. ${ }^{48}$ Predominant elevation of ALT and AST indicates hepatocellular injury. ${ }^{12}$ The abnormalities in AST have been more severe compared with those of ALT. ${ }^{20,22,23,34,37,44,45}$ This finding is intriguing, as ALT, being more liver-specific, is the enzyme that is generally expected to be significantly elevated in case of hepatocellular injury. However, a few studies have hypothesised that AST elevation could be secondary to viral-mediated direct liver damage. ${ }^{20,21}$ The mechanism behind predominant AST elevation in the presence of viral aetiology remains unclear.

Elevation of the ductal enzymes gammaglutamyl transferase and alkaline phosphatase (ALP) has been reported in some studies. 22,23,70 Elevated ALP was also reported alongside elevated AST and ALT in a case of acute hepatitis following COVID-19 infection. ${ }^{61}$ Cardoso et $\mathrm{al}^{71}$ studied the temporal patterns of liver enzyme levels in critically ill patients and observed that a cholestatic pattern emerged later in the course of illness. Most studies have not mentioned the presence of any liver enzyme abnormalities at the time of liver injury. Hence, the extent of liver damage and the pattern of injury could not be accurately assessed. 
TABLE 3. Summary of studies without pre-defined criteria for liver injury/hepatic dysfunction in COVID-19

\begin{tabular}{|c|c|c|c|c|c|c|c|}
\hline Author & Study type & $\begin{array}{l}\text { Study } \\
\text { location }\end{array}$ & $\begin{array}{l}\text { Sample } \\
\text { size }\end{array}$ & $\begin{array}{l}\text { Incidence of } \\
\text { liver injury/ } \\
\text { hepatic } \\
\text { dysfunction, } \\
\% \text { (n) }\end{array}$ & $\begin{array}{l}\text { Liver function tests } \\
\text { (median }[\mathrm{IQR}] / \text { mean } \\
\text { [SD]) }\end{array}$ & $\begin{array}{l}\text { Risk factors of } \\
\text { liver injury/hepatic } \\
\text { dysfunction }\end{array}$ & Study notes \\
\hline $\begin{array}{l}\text { Cholankeril } \\
\text { et } \mathrm{al}^{21}\end{array}$ & $\begin{array}{l}\text { Retrospective } \\
\text { observational }\end{array}$ & $\begin{array}{l}\text { California, } \\
\text { United States }\end{array}$ & 116 & $40 \%(26$ of 65$)$ & $\begin{array}{l}\text { ALT }=32(22-48)^{\star} \\
\text { AST }=35(22-58)^{\star} \\
\text { TBIL }=6.84(5.13-11.97)^{\star}\end{array}$ & - & $\begin{array}{l}\text { Severity of COVID-19 was correlated } \\
\text { with elevated AST }\end{array}$ \\
\hline Wan et $\mathrm{al}^{34}$ & Case series & $\begin{array}{l}\text { Chongqing, } \\
\text { China }\end{array}$ & 135 & - & $\begin{array}{l}\mathrm{ALT}=26(12.9-33.15)^{\star} \\
\mathrm{AST}=33.4(27.8-43.7)^{\star} \\
\mathrm{TBIL}=8.6(5.9-13.7)^{\star}\end{array}$ & - & $\begin{array}{l}\text { Patients with severe disease had } \\
\text { higher AST levels }\left(33.6[25.7-44.2]^{*} \mathrm{U} / \mathrm{L}\right) \\
\text { than patients with non-severe disease } \\
\left(22.4[16.9-30.5]^{*} \mathrm{U} / \mathrm{L}\right)\end{array}$ \\
\hline $\begin{array}{l}\text { Zhang et } \\
\mathrm{al}^{29}\end{array}$ & $\begin{array}{l}\text { Retrospective } \\
\text { observational }\end{array}$ & Wuhan, China & 82 & $78 \%(64)$ & $\begin{array}{l}\mathrm{ALT}=30.5(22.0-102.5)^{\star} \\
\mathrm{AST}=74.5(35.5-184.0)^{\star}\end{array}$ & - & $\begin{array}{l}\text { There was a significant correlation } \\
\text { between AST, ALT, and time from initial } \\
\text { symptom to death }\end{array}$ \\
\hline Ding et $\mathrm{al}^{13}$ & $\begin{array}{l}\text { Retrospective } \\
\text { descriptive }\end{array}$ & Wuhan, China & 5 & $60 \%(3)$ & $\begin{array}{l}\mathrm{ALT}=18(7-63)^{\star} \\
\mathrm{AST}=22(15-56)^{\star} \\
\mathrm{TBIL}=5.3(4.3-8)^{\star}\end{array}$ & - & $\begin{array}{l}\text { Liver injury was more commonly seen } \\
\text { in patients with severe COVID-19 }\end{array}$ \\
\hline Du et $\mathrm{al}^{25}$ & $\begin{array}{l}\text { Retrospective } \\
\text { observational }\end{array}$ & Wuhan, China & 85 & $35.3 \%(30)$ & $\begin{array}{l}\mathrm{ALT}=72.9(199.5) \dagger \\
\mathrm{AST}=94.4(263.3) \dagger \\
\mathrm{TBIL}=18.44(13.61) \dagger\end{array}$ & - & - \\
\hline Fu et $\mathrm{al}^{15}$ & $\begin{array}{l}\text { Prospective } \\
\text { case-cohort }\end{array}$ & $\begin{array}{l}\text { Wuhan and } \\
\text { Fuyang, China }\end{array}$ & 355 & $28.5 \%(101)$ & $\begin{array}{l}\mathrm{ALT}=23.0(15.0-42.0)^{\star} \\
\mathrm{AST}=29.0(23.0-54.0)^{\star} \\
\mathrm{TBIL}=10.9(8.0-16.2)^{\star}\end{array}$ & $\begin{array}{l}\text { Male sex, old age, } \\
\text { diabetes mellitus, } \\
\text { lymphopoenia }\end{array}$ & $\begin{array}{l}\text { Patients who were critically ill had } \\
\text { higher levels of ALT }\left(33.0[19.0-61.0]^{\star} \mathrm{U} / \mathrm{L}\right) \\
\text { and AST }\left(49.0[35.0-80.0]^{*} \mathrm{U} / \mathrm{L}\right) \text { than } \\
\text { those who were not. Liver injury at an } \\
\text { early stage of disease increased death } \\
\text { risk }\end{array}$ \\
\hline Li et $\mathrm{al}^{14}$ & $\begin{array}{l}\text { Retrospective } \\
\text { observational }\end{array}$ & Beijing, China & 85 & $38.8 \%(33)$ & $\begin{array}{l}\text { ALT }=28.0(19.5-44.5)^{\star} \\
\text { AST }=29.0(20.5-38.5)^{\star} \\
\text { TBIL }=9.2(6.4-12.1)^{\star}\end{array}$ & $\begin{array}{l}\text { Old age, drinking } \\
\text { history, increased CRP, } \\
\text { decreased lymphocyte } \\
\text { count }\end{array}$ & $\begin{array}{l}\text { Liver injury was more commonly seen } \\
\text { in patients with severe COVID-19 }\end{array}$ \\
\hline Li et $\mathrm{al}^{26}$ & $\begin{array}{l}\text { Retrospective } \\
\text { observational }\end{array}$ & Wuhan, China & 25 & $20 \%(5)$ & $\begin{array}{l}\mathrm{ALT}=24(16.5-46)^{\star} \\
\mathrm{AST}=37(29.5-57.5)^{\star}\end{array}$ & - & $\begin{array}{l}\text { In addition to the lungs, the liver was } \\
\text { the third most commonly damaged } \\
\text { organ in deceased patients }\end{array}$ \\
\hline Lian et $\mathrm{al}^{27}$ & $\begin{array}{l}\text { Retrospective } \\
\text { observational }\end{array}$ & $\begin{array}{l}\text { Zhejiang, } \\
\text { China }\end{array}$ & 465 & $13.1 \%(61)$ & $\begin{array}{l}\mathrm{ALT}=27.95(22.49) \dagger \\
\mathrm{AST}=28.37(17.56) \dagger \\
\mathrm{TBIL}=11.52(7.02) \dagger\end{array}$ & - & - \\
\hline Zhou et al ${ }^{35}$ & $\begin{array}{l}\text { Retrospective } \\
\text { observational }\end{array}$ & $\begin{array}{l}\text { Sichuan, } \\
\text { China }\end{array}$ & 366 & - & - & Chronic liver disease & $\begin{array}{l}\text { Chronic liver disease is a risk factor for } \\
\text { the development of severe disease }\end{array}$ \\
\hline Yang et $\mathrm{al}^{19}$ & $\begin{array}{l}\text { Retrospective } \\
\text { observational }\end{array}$ & Wuhan, China & 92 & $16.3 \%(15)$ & $\begin{array}{l}\mathrm{ALT}=27(3-1693)^{\star} \\
\mathrm{AST}=31(11-6000)^{\star} \\
\mathrm{TBIL}=13.6(4.2-439.0)^{\star}\end{array}$ & - & $\begin{array}{l}\text { Liver injury was commonly seen in } \\
\text { deceased patients with COVID-19 }\end{array}$ \\
\hline Yang et $\mathrm{al}^{28}$ & $\begin{array}{l}\text { Retrospective } \\
\text { observational }\end{array}$ & Wuhan, China & 52 & $29 \%(15)$ & - & - & $\begin{array}{l}\text { Liver dysfunction was commonly seen } \\
\text { in critically ill patients with COVID-19. } \\
\text { Survivors' TBIL was lower than that } \\
\text { of non-survivors (13.1 [4.3]† vs } \\
19.5 \text { [11.6]† } \mu \mathrm{mol} / \mathrm{L})\end{array}$ \\
\hline Yang et $\mathrm{a}^{28}$ & $\begin{array}{l}\text { Retrospective } \\
\text { observational }\end{array}$ & Wuhan, China & 52 & $29 \%(15)$ & - & - & $\begin{array}{l}\text { Liver dysfunction was commonly seen } \\
\text { in critically ill patients with COVID-19. } \\
\text { Survivors' TBIL was lower than that } \\
\text { of non-survivors (13.1 [4.3]† vs } 19.5 \\
[11.6] \dagger \mu \mathrm{mol} / \mathrm{L})\end{array}$ \\
\hline Wang et $\mathrm{a}^{37}$ & $\begin{array}{l}\text { Retrospective } \\
\text { observational }\end{array}$ & Wuhan, China & 339 & - & $\begin{array}{l}\mathrm{ALT}=27(17-44)^{\star} \\
\mathrm{AST}=32(23-46)^{\star}\end{array}$ & - & $\begin{array}{l}\text { Patients who died had higher AST } \\
\text { levels than survivors ( } 43[30-68]^{*} \text { vs } \\
29[22-43]^{\star} \text { U/L). There was no } \\
\text { significant difference in ALT levels } \\
\text { between the two groups }\end{array}$ \\
\hline Wu et $\mathrm{al}^{46}$ & $\begin{array}{l}\text { Retrospective } \\
\text { cohort }\end{array}$ & Wuhan, China & 201 & - & $\begin{array}{l}\mathrm{ALT}=31.00(19.75-47)^{\star} \\
\mathrm{AST}=33.00(26-45)^{\star} \\
\mathrm{TBIL}=11.45(9-14.75)^{\star}\end{array}$ & - & $\begin{array}{l}\text { Liver function indices in patients with } \\
\text { ARDS were increased as compared } \\
\text { to those without ARDS, ie, TBIL } \\
\text { (difference: } 1.90 \mu \mathrm{M} ; 95 \% \mathrm{Cl}=0.60 \text { - } \\
3.30 \mu \mathrm{M} ; \mathrm{P}=0.004 \text { ) }\end{array}$ \\
\hline Qi et al ${ }^{60}$ & Case series & Hubei, China & 3 & - & $\begin{array}{l}\mathrm{ALT}=26(19-44)^{\star} \\
\mathrm{AST}=42(41-103)^{\star} \\
\mathrm{TBIL}=40.4(22.2-62.5)^{\star}\end{array}$ & $\begin{array}{l}\text { Decompensated liver } \\
\text { cirrhosis }\end{array}$ & - \\
\hline
\end{tabular}

Abbreviations: 95\% Cl = 95\% confidence interval; ALT = alanine aminotransferase (U/L); ARDS = adult respiratory distress syndrome; AST = aspartate aminotransferase (U/L); COVID-19 = coronavirus disease 2019; CRP = C-reactive protein; $\mathrm{LQR}=$ interquartile range; $\mathrm{SD}=$ standard deviation; TBIL = total bilirubin $(\mu \mathrm{mol} / \mathrm{L})$

* Data are shown as median and IQR (in parentheses)

† Data are shown as mean and SD (in parentheses) 


\section{Histopathological findings of liver injury in COVID-19}

Histopathological findings of autopsied liver samples have provided evidence of direct viral invasion and changes secondary to hypoxia, sepsis, and proinflammatory and pro-coagulant states. Wang et $\mathrm{al}^{11}$ revealed the presence of hepatic apoptosis, occasional bi- or multi-nucleated hepatocytes, mitochondrial swelling, and decreased glycogen granules. These findings strongly suggest direct cytopathic effects of COVID-19 on the liver. Electron microscopy also showed the presence of viral particles. Other non-specific findings have included varying degrees of steatosis, ${ }^{11,12,49-52}$ mild portal lymphocytic infiltration, ${ }^{11,36,50,51}$ mild sinusoidal dilation, ${ }^{36,51,53}$ and inflamed cells within the sinusoids. ${ }^{12}$ However, samples obtained via needle biopsy did not facilitate effective determination of the histology of the ductal epithelium, which carries a higher density of ACE2Rs. ${ }^{51}$ Ductal pathology was highlighted by Lax et $a l,{ }^{52}$ indicating the presence of canalicular cholestasis and mild nuclear pleomorphism of cholangiocytes. Patterns of both massive and focal patchy necrosis were reported in the periportal and centrilobular areas. ${ }^{51,52}$ The authors suggested that sepsis and systemic inflammation might be responsible for acute hepatic necrosis. Furthermore, reverse transcription-polymerase chain reaction of one liver sample was positive for COVID-19. ${ }^{51}$ In that case, an ultrasound-guided autopsy observed centrilobular congestion (which was likely attributable to shock), ischaemic necrosis, portal tract inflammation, and Kupffer cell activation. ${ }^{51}$

The watery degeneration of some hepatocytes observed by Cai et $\mathrm{al}^{12}$ was likely due to ischaemia and hypoxia. The presence of thrombi within the liver, among other organs, also demonstrates the possibility of COVID-associated coagulopathy. ${ }^{52}$ Liver involvement with COVID-19 infection may further elaborate the inflammatory cascade and alter the secretion of coagulation factors, thus playing a role in causing widespread thrombosis. ${ }^{52}$ Endotheliitis, acute and chronic vascular changes, and sinusoidal arterialisation due to pressure elevation observed in the liver further support the involvement of underlying endothelial pathology in causing coagulative derangements. ${ }^{53,69}$

\section{Liver injury as a marker of the severity of COVID-19}

Studies have consistently shown liver injury to be associated with severe COVID-19.11-16,34,42-44 Deranged LFTs have also been linked to prolonged hospital stays ${ }^{18}$ and worse clinical outcomes. ${ }^{19,38,40,45}$ Disease severity is most likely linked with the elevation of AST rather than ALT. ${ }^{20,21,45}$ Additionally, hypoproteinaemia and cholestasis in early-stage disease have been shown to increase the risk of

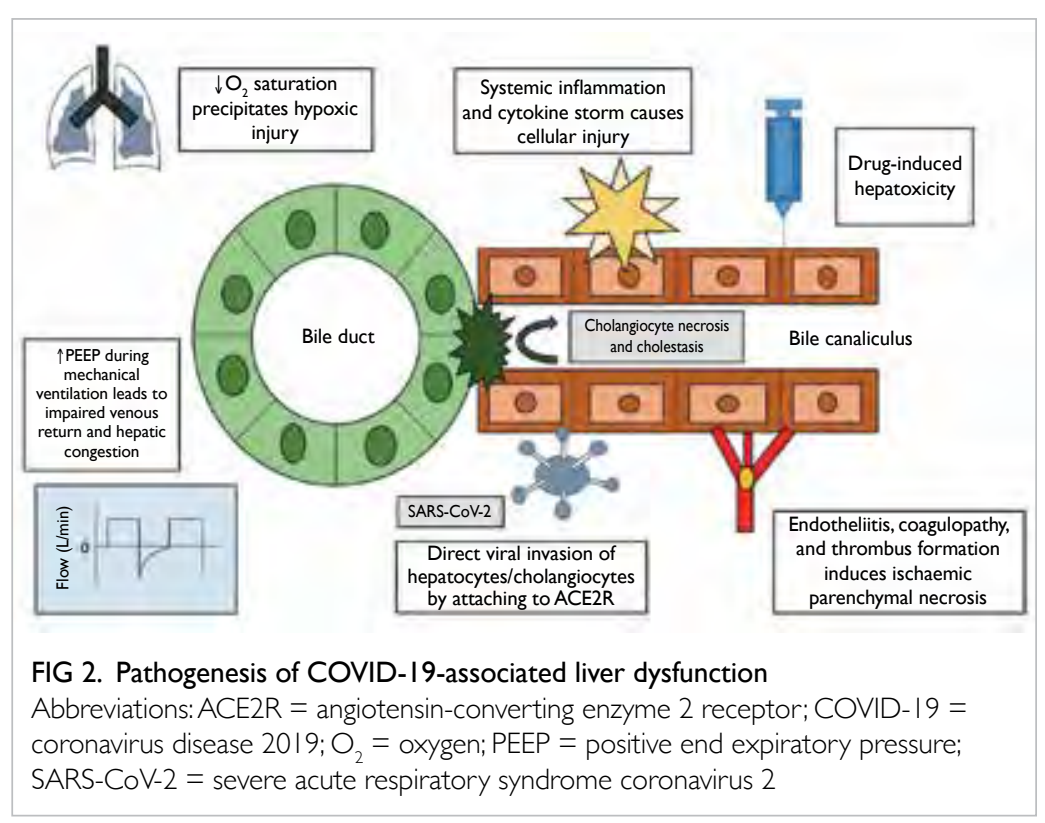

death. ${ }^{15}$ However, the impact of AST on mortality has been controversial. ${ }^{22,46}$

These findings cannot reliably establish that elevated LFT levels were solely caused by COVID-19 infection, as many studies did not exclude patients with CLD, nor did they consider other possible reasons for liver enzyme elevation. Furthermore, there is still not enough evidence to suggest that mild derangement has a high likelihood of progressing into fulminant liver failure. Yet, patients with deranged LFT patterns of the hepatocellular or mixed types at the time of admission or during hospitalisation were more likely to progress to severe disease, ${ }^{12,47}$ thus necessitating adequate monitoring. ${ }^{38,44,45,48}$ Additionally, there is no evidence that liver dysfunction can directly cause mortality in patients with COVID-19.

\section{Risk factors for liver injury in COVID-19}

Studies have reported associations between multiple risk factors and liver injury in the setting of SARS-CoV-2 infection:

1. Abnormal white blood cell parameters, including elevated neutrophils and decreased lymphocytes, have been associated with elevated risk of liver injury. ${ }^{14,15,17,22}$ The loss of lymphocytes responsible for suppression of the immune response during viral infection may have contributed to the damage. ${ }^{14}$ Similarly, high levels of C-reactive protein and procalcitonin were associated with increased risk of liver damage. ${ }^{14,17,18}$ The cytokine storm and systemic inflammation might be implicated, as they result in leukocyte activation and the release of a large quantity of inflammatory mediators that directly or indirectly damage cells. $^{14}$

2. The use of hepatotoxic drugs, including antivirals, 
hydroxychloroquine, tocilizumab (discussed below), ${ }^{12,16,18,58}$ and antifungals for superimposed infections has been established as a risk factor for liver dysfunction. ${ }^{22}$ Systemic corticosteroids were also associated with an increased risk of AST elevation, ${ }^{22}$ perhaps due to drug-induced lymphopoenia and alteration of the immune response.

3. A correlation between the severity of lung involvement and the incidence of liver injury has also been noticed. ${ }^{17}$ As severe lung lesions indicate a robust inflammatory state, the liver might be affected for the same reason (ie, a hyperinflammatory state). ${ }^{17}$ That study did not indicate the role of hypoxia, which is also a possible contributing factor to hepatic damage.

4. Non-modifiable risk factors such as male sex (odds ratio $=1.60 ; \mathrm{P}<0.001$ ) and old age (odds ratio=1.01; $\mathrm{P}=0.031$ ) have been linked to a higher risk of liver damage. ${ }^{22,23}$

5. Patients with elevated ALT levels were more likely to have a history of drinking $(\mathrm{P}=0.032) .{ }^{14}$ However, that study did not comment on elevation of AST, the dominant enzyme involved in both alcoholic liver disease and COVID-19. Furthermore, that study's very small sample size necessitates further investigation of this risk factor.

6. Patients with gastrointestinal symptoms were more likely to have liver injury than those without such symptoms $(\mathrm{P}=0.035) .{ }^{24}$

7. Diabetes mellitus was a risk factor for cholestasis in patients with COVID-19 $(\mathrm{P}=0.044),{ }^{15}$ which is predicted to be a possible mechanism of liver injury in the setting of viral infection..$^{65}$

8. Invasive mechanical ventilation increased the risk of LFT elevation ${ }^{42}$ and acute liver injury. ${ }^{33}$ Such injury may be caused by hepatic congestion that results from elevated right atrial pressure, which in turn is caused by high levels of positive end expiratory pressure. ${ }^{68}$

\section{Drug-induced hepatotoxicity in COVID-19}

The drugs currently used to manage COVID-19 infection also carry hepatotoxic potential. Muhović et $\mathrm{al}^{56}$ reported a 40 -fold rise in transaminases following two doses of tocilizumab, an interleukin-6 receptor antagonist, which regressed 10 days later. Morena et $\mathrm{al}^{59}$ also reported elevated liver enzymes in $29 \%$ of patients who were receiving tocilizumab. In addition, Falcão et $\mathrm{a}^{58}$ reported a 10 -fold elevation in transaminases following two doses of hydroxychloroquine. Upon withdrawal, the enzyme levels dropped to near normal after 5 days.

Antivirals have also been demonstrated to cause liver toxicity. In one study, people receiving lopinavir/ritonavir had a higher incidence of liver dysfunction compared with those in whom these drugs were not administered $(51.8 \%$ vs $31.3 \%$, respectively). ${ }^{18}$ Similarly, Young et $\mathrm{al}^{55}$ reported abnormal LFTs in three out of five patients receiving lopinavir/ritonavir. According to Cai et al, ${ }^{12}$ the use of lopinavir/ritonavir increased the likelihood of liver injury 4-fold. Durante-Mangoni et a ${ }^{54}$ reported that remdesivir caused elevation of liver enzymes in three out of four patients, and Weber et $\mathrm{al}^{57}$ suggested that drugs may play a role in precipitating acute liver failure. Administration of lopinavir/ritonavir and interferon was followed by progressive worsening of LFTs. This effect may have been attenuated by the use of Ramipril for arterial hypertension. ${ }^{57}$ Additionally, Lei et $\mathrm{al}^{22}$ showed that elevated AST and ALP levels were associated with the use of antifungal medications. The above findings are from case reports, retrospective studies, and very small-scale prospective studies. Further large-scale prospective studies, including randomised controlled trials, need to be conducted to establish their efficacy and safety in patients with COVID-19.

\section{Chronic liver disease and COVID-19}

The effects of underlying liver disease on the severity of COVID-19 are controversial. Zhou et a ${ }^{35}$ suggested the presence of CLD as a risk factor for severe COVID-19. However, that study included only eight known cases of CLD. Similarly, Qi et al ${ }^{60}$ indicated that decompensated liver cirrhosis might be a risk factor for poor outcomes of COVID-19. In contrast, a meta-analysis by Wang et $\mathrm{al}^{72}$ that included five studies concluded that prior liver disease does not impact the severity of COVID-19. Likewise, the presence of pre-existing cirrhosis had no direct prognostic association in the setting of COVID-19. ${ }^{73}$ Some studies in our review included patients with CLD, which may account for some of the LFT derangements observed in patients. A meta-analysis by Mantovani et $\mathrm{al}^{41}$ estimated that the baseline prevalence of CLD was $3 \%$. This figure is much lower than the proportion of people with liver dysfunction. Hence, the role of CLD in worsening the prognosis of COVID-19 infection seems to be minor, if there is any.

Nevertheless, acute-on-chronic liver failure (ACLF) following COVID-19 infection has been reported. One case was a female patient with decompensated alcoholic cirrhosis (ACLF Grade 2) who developed a mixed hepatic and cholestatic pattern of liver dysfunction following COVID-19 infection. However, her prognosis was good. ${ }^{74}$ Another patient was an older man with ACLF Grade 1 non-alcoholic cirrhosis. He developed hepatorenal syndrome-type acute kidney injury following COVID-19 infection. His liver failure subsequently progressed to Grade 2 after catheterassociated urinary tract infections and complicated paracentesis. ${ }^{75}$ 


\section{Oro-faecal transmission and liver injury}

Cui et $\mathrm{al}^{61}$ revealed that anal swabs of an infant with liver injury remained positive for COVID-19 even after throat swabs returned to a negative state. However, polymerase chain reaction of stool samples was not performed in most of the articles included in our review. The association of liver dysfunction with the risk of oro-faecal transmission remains to be investigated. If transmission via this route is possible, existing isolation and discharge protocols may need to be revised.

\section{Limitations and recommendations}

Our review is subject to certain limitations. First, the majority of the included studies did not have any specific pre-defined clinical criteria for diagnosing liver injury in COVID-19. Moreover, the included studies did not distinguish between a history of liver disease (eg, CLD) and liver injury secondary to COVID-19. Hence, our results need to be interpreted cautiously, as they do not accurately describe the level of incidence of liver injury that is caused by COVID-19. Second, we did not include studies published in any language other than English, which might have provided additional insight. Third, the inclusion of a large number of studies prevented us from critically appraising the individual studies' sources of evidence. There is a need for a comprehensive systematic review or meta-analysis to summarise the statistics and provide a clearer picture of liver injury in SARS-CoV-2 infection. Transplant recipients, a group that is vulnerable to liver injury, were also not reviewed.

Many studies have defined 'liver injury' as non-specific elevation of LFTs above the upper limit of normal. Further, many of the investigated studies did not assess the bilirubin levels or coagulation profiles of patients with COVID-19, both of which are important indicators of liver function. Moreover, there have been no reports of liver failure or hepatic cell death secondary to COVID-19 to date. Hence, we recommend the use of scientifically relevant terms 'liver dysfunction' or 'liver enzyme derangement' to explain non-specific LFT abnormalities until an appropriate definition for liver injury is devised. Furthermore, we propose that pre-defined criteria for liver injury should be set and that mild, nonspecific derangements of liver function should not be labelled as liver injury. Given the existing controversy in the literature, we recommend a thorough investigation into the pathogenesis of liver injury, especially the mode of direct viral invasion.

Additional studies are required to investigate whether mild derangement of liver function can cause hepatic failure in COVID-19. The reason for the hepatocellular pattern with predominant AST elevation also needs to be elucidated. Finally, the safety and efficacy of hepatotoxic drugs in COVID-19 should also be established via randomised controlled trials.

\section{Conclusion}

Liver injury is a common extrapulmonary feature of COVID-19. However, the absence of standardised clinical criteria for liver injury in this setting needs to be addressed. Derangements of LFT levels are markers of the severity of COVID-19 infection, but the association between LFT derangements and disease progression requires further investigation because to date, liver dysfunction has not been shown to directly cause mortality in patients with COVID-19. The pattern of injury is predominantly hepatocellular, accompanied by greater elevation of AST than of ALT. Possible pathogenetic mechanisms include direct viral invasion, hypoxia, systemic inflammation, endothelial dysfunction, and the use of mechanical ventilation. Histopathological findings in the liver support viral-induced pathology in addition to non-specific changes. Nevertheless, these studies are sparse, and more research is required. Potentially hepatotoxic drugs have also been observed to cause liver injury in patients with COVID-19, and thus, the administration of these drugs necessitates careful monitoring. Large-scale studies are needed to establish their role in the management of COVID-19.

\section{Author contributions}

Concept or design: T Bin Arif.

Acquisition of data: T Bin Arif, S Khalid, MS Siddiqui, H Hussain.

Analysis or interpretation of data: H Sohail.

Drafting of the manuscript: S Khalid, MS Siddiqui, H Hussain. Critical revision of the manuscript for important intellectual content: T Bin Arif, H Sohail.

All authors had full access to the data, contributed to the study, approved the final version for publication, and take responsibility for its accuracy and integrity.

\section{Conflicts of interest}

All authors have disclosed no conflicts of interest.

\section{Funding/support}

This research received no specific grant from any funding agency in the public, commercial, or not-for-profit sectors.

\section{References}

1. World Health Organization. Coronavirus disease (COVID-2019) situation reports-124. Available from: https://www.who.int/docs/default-source/coronaviruse/ situation-reports/20200523-covid-19-sitrep-124. pdf?sfvrsn=9626d639_2. Accessed 29 May 2020.

2. Wang W, Xu Y, Gao R, et al. Detection of SARS-CoV-2 in different types of clinical specimens. JAMA 2020;323:18434.

3. Chen J, Zhu H, Wang D, et al. Clinical features of stool SARS-CoV-2 RNA positive in 137 COVID-19 patients in 
Taizhou, China. SSRN Electronic J 2020 Mar 23. Available from: https://papers.ssrn.com/sol3/papers.cfm?abstract_ $\mathrm{id}=3551383$. Accessed 28 May 2020.

4. Pan L, Mu M, Yang $\mathrm{P}$, et al. Clinical characteristics of COVID-19 patients with digestive symptoms in Hubei, China: a descriptive, cross-sectional, multicenter study. Am J Gastroenterol 2020;115:766-73.

5. Chen T, Wu D, Chen $\mathrm{H}$, et al. Clinical characteristics of 113 deceased patients with coronavirus disease 2019: retrospective study. BMJ 2020;368:m1091.

6. Chen N, Zhou M, Dong X, et al. Epidemiological and clinical characteristics of 99 cases of 2019 novel coronavirus pneumonia in Wuhan, China: a descriptive study. Lancet 2020;395:507-13.

7. Zhang C, Shi L, Wang FS. Liver injury in COVID-19: management and challenges. Lancet Gastroenterol Hepatol 2020;5:428-30.

8. Mehta P, McAuley DF, Brown M, et al. COVID-19: consider cytokine storm syndromes and immunosuppression. Lancet 2020;395:1033-4.

9. Arksey H, O’Malley L. Scoping studies: towards a methodological framework. Int J Soc Res Methodol 2005;8:19-32.

10. Tricco AC, Lillie E, Zarin W, et al. PRISMA extension for scoping reviews (PRISMA-ScR): checklist and explanation. Ann Inter Med 2018;169:467-73.

11. Wang Y, Liu S, Liu $\mathrm{H}$, et al. SARS-CoV-2 infection of the liver directly contributes to hepatic impairment in patients with COVID-19. J Hepatol 2020;73:807-16.

12. Cai Q, Huang D, Yu H, et al. COVID-19: Abnormal liver function tests. J Hepatol 2020;73:566-74.

13. Ding Q, Lu P, Fan Y, Xia Y, Liu M. The clinical characteristics of pneumonia patients coinfected with 2019 novel coronavirus and influenza virus in Wuhan, China. J Med Virol 2020 Mar 20. Epub ahead of print.

14. Li L, Li S, Xu M, et al. Risk factors related to hepatic injury in patients with corona virus disease 2019. medRxiv. 2020 Mar 10. Available from: https://www.medrxiv.org/content/ 10.1101/2020.02.28.20028514v2. Accessed 27 May 2020.

15. Fu L, Fei J, Xu S, et al. Acute liver injury and its association with death risk of patients with COVID-19: a hospitalbased prospective case-cohort study. medRxiv. 2020 Apr 6. Available from: https://www.medrxiv.org/content/10.1101/ 2020.04.02.20050997v1. Accessed 27 May 2020.

16. Cai Q, Huang D, Ou P, et al. COVID-19 in a designated infectious diseases hospital outside Hubei Province, China. Allergy 2020;75:1742-52.

17. Xie H, Zhao J, Lian N, Lin S, Xie Q, Zhuo H. Clinical characteristics of non-ICU hospitalized patients with coronavirus disease 2019 and liver injury: a retrospective study. Liver Int 2020;40:1321-6.

18. Fan Z, Chen L, Li J, et al. Clinical features of COVID-19. related liver functional abnormality. Clin Gastroenterol Hepatol 2020;18:1561-6.

19. Yang F, Shi S, Zhu J, Shi J, Dai K, Chen X. Analysis of 92 deceased patients with COVID-19. J Med Virol 2020 Apr 15. Epub ahead of print.

20. Bloom PP, Meyerowitz EA, Reinus Z, et al. Liver biochemistries in hospitalized patients with COVID-19. Hepatology 2020 May 16. Epub ahead of print.

21. Cholankeril G, Podboy A, Aivaliotis VI, et al. High prevalence of concurrent gastrointestinal manifestations in patients with SARS-CoV-2: early experience from California. Gastroenterology 2020;159:775-7.

22. Lei F, Liu YM, Zhou F, et al. Longitudinal association between markers of liver injury and mortality in COVID19 in China. Hepatology 2020;72:389-98.

23. Hajifathalian K, Krisko T, Mehta A, et al. Gastrointestinal and hepatic manifestations of 2019 novel coronavirus disease in a large cohort of infected patients from New York: clinical implications. Gastroenterology 2020;159:1137-40. e2.

24. Jin $\mathrm{X}$, Lian JS, $\mathrm{Hu} \mathrm{JH}$, et al. Epidemiological, clinical and virological characteristics of 74 cases of coronavirusinfected disease 2019 (COVID-19) with gastrointestinal symptoms. Gut 2020;69:1002-9.

25. Du Y, Tu L, Zhu P, et al. Clinical features of 85 fatal cases of COVID-19 from Wuhan: a retrospective observational study. Am J Respir Crit Care Med 2020;201:1372-9.

26. Li X, Wang L, Yan S, et al. Clinical characteristics of 25 death cases with COVID-19: a retrospective review of medical records in a single medical center, Wuhan, China. Int J Infect Dis 2020;94:128-32.

27. Lian J, Jin X, Hao S, et al. Epidemiological, clinical, and virological characteristics of 465 hospitalized cases of coronavirus disease 2019 (COVID-19) from Zhejiang province in China. Influenza Other Respir Viruses 2020;14:564-74.

28. Yang X, Yu Y, Xu J, et al. Clinical course and outcomes of critically ill patients with SARS-CoV-2 pneumonia in Wuhan, China: a single-centered, retrospective, observational study. Lancet Respir Med 2020;8:475-81.

29. Zhang B, Zhou X, Qiu Y, et al. Clinical characteristics of 82 death cases with COVID-19. medRxiv. 2020 Feb 27. Available from: https://www.medrxiv.org/content/10.1101/ 2020.02.26.20028191v1. Accessed 28 May 2020.

30. Zhu J, Ji P, Pang J, et al. Clinical characteristics of 3062 COVID-19 patients: a meta-analysis. J Med Virol $2020 \mathrm{Apr}$ 15. Epub ahead of print.

31. Chen G, Wu D, Guo W, et al. Clinical and immunological features of severe and moderate coronavirus disease 2019. J Clin Invest 2020;130:2620-9.

32. Qi X, Liu C, Jiang Z, et al. Multicenter analysis of clinical characteristics and outcome in patients with COVID-19 who develop liver injury. J Hepatol 2020;73:455-8.

33. Zheng Y, Sun L, Xu M, et al. Clinical characteristics of 34 COVID-19 patients admitted to ICU in Hangzhou, China. medRxiv. 2020 Apr 15. Available from: https://www. medrxiv.org/content/10.1101/2020.04.12.20062604v1. Accessed 28 May 2020.

34. Wan S, Xiang Y, Fang W, et al. Clinical features and treatment of COVID-19 patients in Northeast Chongqing. J Med Virol 2020;92:797-806.

35. Zhou Y, He Y, Yang H, et al. Development and validation a nomogram for predicting the risk of severe COVID-19: a multi-center study in Sichuan, China. PLoS One 2020;15:e0233328.

36. Zhang Y, Zheng L, Liu L, Zhao M, Xiao J, Zhao Q. Liver impairment in COVID-19 patients: a retrospective analysis of 115 cases from a single centre in Wuhan City, China. Liver Int 2020;40:2095-103.

37. Wang L, He W, Yu X, et al. Coronavirus disease 2019 in elderly patients: characteristics and prognostic factors based on 4-week follow-up. J Infect 2020;80:639-45.

38. Parohan M, Yaghoubi S, Seraj A. Liver injury is associated with severe coronavirus disease 2019 (COVID-19) infection: a systematic review and meta-analysis of retrospective studies. Hepatol Res 2020;50:924-35.

39. Mao R, Qiu Y, He JS, et al. Manifestations and prognosis of gastrointestinal and liver involvement in patients with COVID-19: a systematic review and meta-analysis. Lancet Gastroenterol Hepatol 2020;5:667-78.

40. Henry BM, de Oliveira MH, Benoit S, Plebani M, Lippi G. Hematologic, biochemical and immune biomarker abnormalities associated with severe illness and mortality in coronavirus disease 2019 (COVID-19): a meta-analysis. 
Clin Chem Lab Med 2020;58:1021-8.

41. Mantovani A, Beatrice G, Dalbeni A. Coronavirus disease 2019 and prevalence of chronic liver disease: a metaanalysis. Liver Int 2020;40:1316-20.

42. Israelsen SB, Kristiansen KT, Hindsberger B, et al. Characteristics of patients with COVID-19 pneumonia at Hvidovre Hospital, March-April 2020. Dan Med J 2020;67:A05200313.

43. Zhao X, Lei Z. The impact of coronavirus disease 2019 (COVID-19) on liver injury in China: a systematic review and meta-analysis. medRxiv. 2020 May 8. Available from: https://www.medrxiv.org/content/10.1101/2020.05.03.200 89557v1. Accessed 28 May 2020.

44. Dong X, Zeng DY, Cai YY, et al. Liver chemistries in patients with severe or non-severe COVID-19: a metaanalysis. medRxiv. 2020 Apr 29. Available from: https:// www.medrxiv.org/content/10.1101/2020.04.24.2007417 9v1. Accessed 29 May 2020.

45. Xing QQ, Dong X, Ren YD, et al. Liver chemistries in COVID-19 patients with survival or death: a metaanalysis. medRxiv. 2020 May 1. Available from: https:// www.medrxiv.org/content/10.1101/2020.04.26.2008058 0v1. Accessed 28 May 2020.

46. Wu C, Chen X, Cai Y, et al. Risk factors associated with acute respiratory distress syndrome and death in patients with coronavirus disease 2019 pneumonia in Wuhan, China. JAMA Intern Med 2020;180:1-11.

47. Xu L, Mao Y, Chen G. Risk factors for severe corona virus disease 2019 (COVID-19) patients: a systematic review and meta analysis. medRxiv. 2020 Apr 1. Available from: https://www.medrxiv.org/content/10.1101/2020.03.30.200 47415v1. Accessed 28 May 2020.

48. Chen C, Jiang J, Xu X, Hu Y, Hu Y, Zhao Y. Dynamic liver function indexes monitoring and clinical characteristics in three types of COVID-19 patients. medRxiv. 2020 May 20. Available from: https://www.medrxiv.org/content/10.1101/ 2020.05.13.20099614v2. Accessed 28 May 2020.

49. Nunes Duarte-Neto A, de Almeida Monteiro RA, da Silva LF, et al. Pulmonary and systemic involvement of COVID-19 assessed by ultrasound-guided minimally invasive autopsy. Histopathology 2020 May 22. Epub ahead of print.

50. Xu Z, Shi L, Wang Y, et al. Pathological findings of COVID-19 associated with acute respiratory distress syndrome. Lancet Respir Med 2020;8:420-2.

51. Tian S, Xiong Y, Liu H, et al. Pathological study of the 2019 novel coronavirus disease (COVID-19) through postmortem core biopsies. Modern Pathol 2020;33:100714.

52. Lax SF, Skok K, Zechner P, et al. Pulmonary arterial thrombosis in COVID-19 with fatal outcome: results from a prospective, single-center, clinicopathologic case series. Ann Intern Med 2020 May 14. Epub ahead of print.

53. Sonzogni A. Liver histopathology in COVID 19 infection is suggestive of vascular alteration. 2020 May 11. Available from: https://www.medrxiv.org/content/10.1101/2020.05. 06.20092718v1. Accessed 28 May 2020.

54. Durante-Mangoni E, Andini R, Bertolino L, et al. Early experience with remdesivir in SARS-CoV-2 pneumonia. Infection 2020;48:779-82.

55. Young BE, Ong SW, Kalimuddin S, et al. Epidemiologic features and clinical course of patients infected with SARSCoV-2 in Singapore. JAMA 2020;323:1488-94.

56. Muhović D, Bojović J, Bulatović A, et al. First case of druginduced liver injury associated with the use of tocilizumab in a patient with COVID-19. Liver Int 2020 May 17. Epub ahead of print.

57. Weber S, Mayerle J, Irlbeck M, Gerbes AL. Severe liver failure during SARS-CoV-2 infection. Gut 2020;69:1365-7.

58. Falcão MB, Pamplona de Góes Cavalcanti L, Filgueiras Filho NM, Antunes de Brito CA. Case report: hepatotoxicity associated with the use of hydroxychloroquine in a patient with COVID-19. Am J Trop Med Hyg 2020;102:1214-6.

59. Morena V, Milazzo L, Oreni L, et al. Off-label use of tocilizumab for the treatment of SARS-CoV-2 pneumonia in Milan, Italy. Eur J Intern Med 2020;76:36-42.

60. Qi X, Wang J, Li X, et al. Clinical course of COVID-19 in patients with pre-existing decompensated cirrhosis: initial report from China. Hepatol Int 2020;14:478-82.

61. Wander P, Epstein M, Bernstein D. COVID-19 presenting as acute hepatitis. Am J Gastroenterol 2020;115:941-2.

62. Cui Y, Tian M, Huang D, et al. A 55-day-old female infant infected with 2019 novel coronavirus disease: presenting with pneumonia, liver injury, and heart damage. J Infect Dis 2020;221:1775-81.

63. Chai X, Hu L, Zhang Y, et al. Specific ACE2 expression in cholangiocytes may cause liver damage after 2019-nCoV infection. bioRxiv. 2020 Feb 4. Available from: https:// www.biorxiv.org/content/10.1101/2020.02.03.931766v1. Accessed 28 May 2020.

64. Seow JJ, Pai R, Mishra A, et al. scRNA-seq reveals ACE2 and TMPRSS2 expression in TROP2+ liver progenitor cells: implications in COVID-19 associated liver dysfunction. bioRxiv. 2020 Mar 25. Available from: https://www.biorxiv. org/content/10.1101/2020.03.23.002832v1. Accessed 28 May 2020.

65. Zhao B, Ni C, Gao R, et al. Recapitulation of SARS-CoV-2 infection and cholangiocyte damage with human liver ductal organoids. Protein Cell 2020;11:771-5.

66. Zhou L, Niu Z, Jiang X, et al. Systemic analysis of tissue cells potentially vulnerable to SARS-CoV-2 infection by the protein-proofed single-cell RNA profiling of ACE2, TMPRSS2 and Furin proteases. 2020 Apr 18. Available from: https://www.biorxiv.org/content/10.1101/2020.04.0 6.028522v2. Accessed 28 May 2020.

67. Waseem N, Chen PH. Hypoxic hepatitis: a review and clinical update. J Clin Transl Hepatol 2016;4:263-8.

68. Kukla M, Skonieczna-Żydecka K, Kotfis K, et al. COVID-19, MERS and SARS with concomitant liver injury-systematic review of the existing literature. J Clin Med 2020;9:1420.

69. Varga Z, Flammer AJ, Steiger P, et al. Endothelial cell infection and endotheliitis in COVID-19. Lancet 2020;395:1417-8.

70. Tian S, Zhu X, Sun X, et al. Longitudinal analysis of laboratory findings during the process of recovery for patients with COVID-19. medRxiv. 2020 Apr 7. Available from: https://www.medrxiv.org/content/10.1101/2020.04. 04.20053280v1. Accessed 28 May 2020.

71. Cardoso FS, Pereira R, Germano N. Liver injury in critically ill patients with COVID-19: a case series. Crit Care 2020;24:190.

72. Wang B, Li R, Lu Z, Huang Y. Does comorbidity increase the risk of patients with COVID-19: evidence from metaanalysis. Aging (Albany NY) 2020;12:6049-57.

73. Qi X, Liu Y, Wang J, et al. Clinical course and risk factors for mortality of COVID-19 patients with pre-existing cirrhosis: a multicentre cohort study. Gut 2020 May 20. Epub ahead of print.

74. Qiu H, Wander P, Bernstein D, Satapathy SK. Acute on chronic liver failure from novel severe acute respiratory syndrome coronavirus 2 (SARS-CoV-2). Liver Int 2020;40:1590-3.

75. Große K, Kramer M, Trautwein C, Bruns T. SARS-CoV-2 as an extrahepatic precipitator of acute-on-chronic liver failure. Liver Int 2020;40:1792-3. 